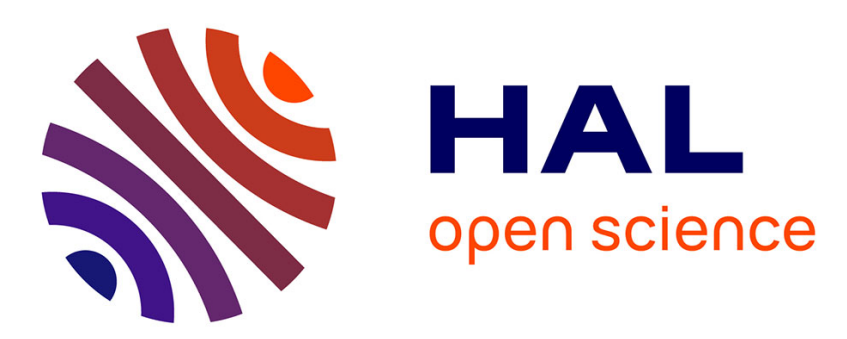

\title{
Etude de la cinétique d'agrégation érythrocytaire dans un écoulement de Couette
}

P. Mills, D. Quemada, J. Dufaux

\section{To cite this version:}

P. Mills, D. Quemada, J. Dufaux. Etude de la cinétique d'agrégation érythrocytaire dans un écoulement de Couette. Revue de Physique Appliquée, 1980, 15 (8), pp.1357-1366. 10.1051/rphysap:019800015080135700 . jpa-00244859

\section{HAL Id: jpa-00244859 https://hal.science/jpa-00244859}

Submitted on 1 Jan 1980

HAL is a multi-disciplinary open access archive for the deposit and dissemination of scientific research documents, whether they are published or not. The documents may come from teaching and research institutions in France or abroad, or from public or private research centers.
L'archive ouverte pluridisciplinaire HAL, est destinée au dépôt et à la diffusion de documents scientifiques de niveau recherche, publiés ou non, émanant des établissements d'enseignement et de recherche français ou étrangers, des laboratoires publics ou privés. 


\title{
Etude de la cinétique d'agrégation érythrocytaire dans un écoulement de Couette
}

\author{
P. Mills, D. Quemada et J. Dufaux \\ Laboratoire de Biorhéologie et d'Hydrodynamique Physiologique (*), \\ (I.E.R. de Physique, Université Paris VII, 2, place Jussieu, 75221 Paris Cedes 05, France \\ (Rę̧u le 16 janvier 1980, révisé le 16 avril 1980, accepté le 28 avril 1980)
}

\begin{abstract}
Résumé. - Etude de la cinétique d'agrégation érythrocytaire dans un écoulement de Couette.
Une théorie de la lumière transmise et rétrodiffusée par ủne suspension sanguine a été étudiée en tenant compte de l'agrégation et de l'orientation des particules dans un écoulement de Couette.

Les résultats théoriques permettent d'interpréter quantitativement les mesures expérimentales de la lumière laser rétrodiffusée par une suspension sanguine dans un écoulement de Couette. On propose une relation entre la taille des rouleaux et le taux de cisaillement moyen.
\end{abstract}

\begin{abstract}
Study of blood suspension aggregation in a Couette flow.
Transmitted and backscattered light by blood suspension was theoretically studied, taking in account aggregation and orientation in a Couette flow.

Theory can bring quantitative explanations of experimental data about laser light backscattered by blood suspension in a Couette flow.

Relation between rouleaux size and the mean shear rate is proposed.
\end{abstract}

1. Introduction. - L'agrégation des cellules est certainement le plus important des facteurs entraînant un comportement non newtonien du sang humain normal. Les cellules empilées forment des rouleaux; cette agrégation est réversible : les rouleaux sont facilement détruits sous l'action d'une force extérieure et se reforment lorsque l'action de cette force extérieure cesse. Au repos, les rouleaux forment un réseau tridimensionnel. Quand une contrainte est appliquée à la suspension, le réseau est brisé en rouleaux individuels dont la dimension est d'autant plus petite que le taux.de cisaillement est grand. A chaque valeur du taux de cisaillement on peut associer une valeur moyenne de la taille des agrégats correspondant à un équilibre dynamique atteint après un temps fonction des paramètres décrivant les interactions entre les particules. La rhéologie des suspensions de particules agrégeables comme les hématies du sang humain normal requiert la connaissance du processus de formation des rouleaux. C'est pourquoi nous nous sommes intéressés à une méthode expérimentale qui

$\left(^{*}\right)$ Equipe de Recherche Associée du C.N.R.S. permet de relier le flux lumineux rétrodiffusé par les rouleaux à leur taille moyenne.

Le fait que la lumière transmise à travers un écoulement sanguin varie avec le débit a été mesuré quantitativement pour la première fois par Kramer (1935). Lorsque les hématies sont en suspension dans un milieu artificiel non agrégeant, les variations de la lumière transmise ou rétrodiffusée sont dues à l'orientation et à la déformation des hématies [1,2]. Lorsque les observations portent sur du sang complet des effets très importants dus à l'agrégation des globules sont observables pour des taux de cisaillement faibles $\left(\dot{\gamma}<50 \mathrm{~s}^{-1}\right)$ [3-8]. Ces observations ne peuvent conduire à des interprétations quantitatives que lorsque l'écoulement est du type Couette. Dans le cas d'un écoulement de Poiseuille, il est plus malaisé de traiter l'information recueillie puisqu'elle résulte de l'intégration des interactions du faisceau lumineux avec différentes couches du fluide pour lesquelles le taux de cisaillement est variable.

Parmi les travaux les plus récents signalons un important travail expérimental effectué par $\mathrm{H}$. SchmidShonbein et son équipe [6] sur le flux lumineux transmis à travers du sang normal ou traité, contenu dans le 
volume de mesure d'un viscosimètre cône-plan et en France le travail d'Healy [5]. Malheureusement, les résultats [6] sont restés qualitatifs. On montre en effet que la lumière transmise à travers une suspension de grosses particules est une fonction assez compliquée de l'épaisseur géométrique du milieu éclairé et des coefficients d'absorption et de diffusion des globules [4]. Par contre, le flux de la lumière rétrodiffusé s'exprime de façon assez simple lorsque le milieu est assez épais pour que le flux transmis soit faible. D'autre part, l'agrégomètre utilisé par Healy, conçu par Dognon [9] est mal adapté à des mesures de rétrodiffusion dans des géométries autres que planes et ne comporte pas de taux de cisaillement contrôlé. Nous pensons avoir considérablement amélioré la mesure par l'emploi comme source lumineuse d'un laser Hélium Néon dont l'émission dans le visible à $\lambda=6328 \AA$ se trouve dans une plage du spectre où l'absorption par les hématies est faible. L'emploi d'une telle source et d'un filtre interférentiel à bande passante étroite permet d'obtenir un remarquable rapport signal/bruit [8].

Nous avons d'autre part amélioré l'interprétation et la quantification du signal de rétrodiffusion grâce à un modèle théorique tenant compte de l'agrégation et de l'orientation des globules [10].

1.1 ETUDE DES FLUX TRANSMIS ET RÉTRODIFFUSÉS. - Nous avons, à partir d'un modèle théorique dû à Shuster [11] et Dognon [4], étudié l'interaction d'un faisceau lumineux avec une suspension de particules susceptibles de s'agréger et pouvant être déformées et orientées dans un écoulement. Considérons une suspension homogène contenue dans un volume délimité par deux plans distants de $l$ (Fig. 1). Soit :

$$
\begin{aligned}
& \varphi_{0}=\text { flux lumineux incident } \\
& \varphi_{\mathrm{r}}=\text { flux lumineux réfléchi } \\
& \varphi_{\mathrm{t}}=\text { flux lumineux transmis }
\end{aligned}
$$

Soit une couche élémentaire d'épaisseur $\mathrm{d} x$ comprise entre $x=0$ et $x=-l$.

$A_{\mathrm{i}}, A_{\mathrm{s}}=$ flux atteignant la couche élémentaire de droite à gauche. ( $A_{\mathrm{i}}$ est le flux isotrope, $A_{\mathrm{s}}$ le flux suivant la direction de $\varphi_{0}$.) $A=A_{\mathrm{i}}+A_{\mathrm{s}}$.

$B_{\mathrm{i}}, B_{\mathrm{s}}=$ flux atteignant la couche élémentaire de gauche à droite $\left(B_{\mathrm{i}}\right.$ est le flux isotrope, $B_{\mathrm{s}}$ le flux suivant la direction de $\left.\varphi_{0}\right) ; B=B_{\mathrm{i}}+B_{\mathrm{s}}$.

$\beta=$ coefficient d'absorption des particules en suspension pour la longueur d'onde $\lambda \mathrm{du}$ faisceau incident,

$$
\begin{aligned}
\mu & =\text { coefficient d'absorption du milieu } \\
& =\beta \frac{\text { volume particulaire }}{\text { volume total }}
\end{aligned}
$$

Soit $f$ le coefficient de diffusion isotrope des particules et $s$ le coefficient de réflexion spéculaire par les

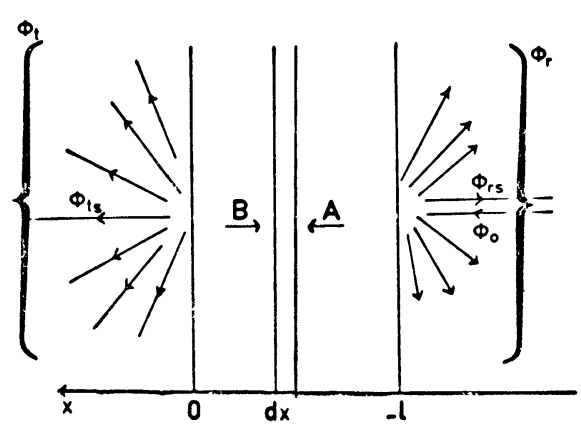

Fig. 1. - Schéma des différents flux lumineux interagissant avec une couche d'épaisseur $l$ de la suspension.

[Diagram showing several luminous fluxes interacting with a suspension layer (thickness $l$ ).]

particules pour une direction donnée du flux incident et pour la longueur d'onde $\lambda$. Le rapport $s / f$ est fonction de la forme et de l'orientation des particules. Il est nul pour des particules sphériques et prend des valeurs grandes pour certaines particules dissymétriques (par exemple pour des particules dont une fraction importante de la surface est plane : $s / f$ sera grand pour des disques et faible pour des cylindres). Dans un écoulement de Couette, ce rapport est une fonction croissante du nombre de particules orientées, donc de la contrainte. $s A_{\mathrm{s}}$ et $s B_{\mathrm{s}}$ représentent les flux réfléchis spéculairement par la couche élémentaire ;

$$
\frac{f}{2} A_{\mathrm{s}} \text { et } \quad \frac{f}{2} B_{\mathrm{s}}
$$

les fractions des flux suivant $\varphi_{0}$ diffusés de façon isotrope. La figure 2 montre qualitativement que la lumière diffusée dans une direction autre que celle

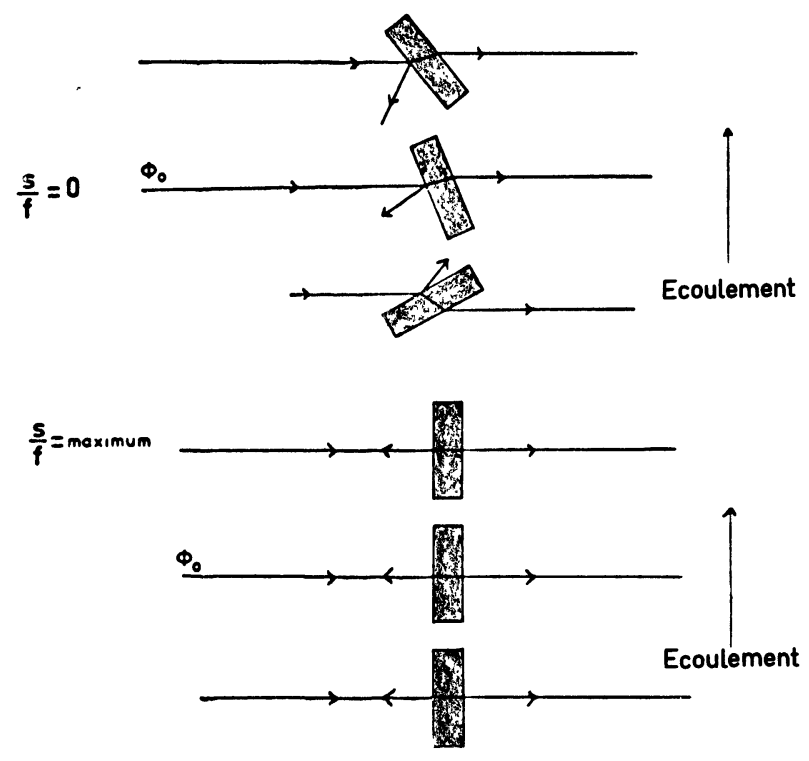

Fig. 2. - Illustration élémentaire des mécanismes de réflexion et de transmission de la lumière suivant l'orientation des particules dans l'écoulement.

[Elementary illustration of light reflection and transmission mechanisms connected with flowing particles orientation.] 
correspondant à la réflexion spéculaire dépend de l'orientation des particules, donc du rapport $s / f$.
Les équations de bilan pour les flux $A_{\mathrm{i}}, A_{\mathrm{s}}, B_{\mathrm{i}}, B_{\mathrm{s}}$ sont :

$$
\mathrm{I}\left\{\begin{array}{l}
\frac{\mathrm{d} A_{\mathrm{s}}}{\mathrm{d} x}=-\left(\mu+\frac{f}{2}+s\right) A_{\mathrm{s}}+s B_{\mathrm{s}} \\
\frac{\mathrm{d} A_{\mathrm{i}}}{\mathrm{d} x}=-\left(\mu+\frac{f}{2}+s\right) A_{\mathrm{i}}+\left(\frac{f}{2}+s\right) B_{\mathrm{i}}+\frac{f}{2} B_{\mathrm{s}} \\
\frac{\mathrm{d} B_{\mathrm{s}}}{\mathrm{d} x}=\left(\mu+\frac{f}{2}+s\right) B_{\mathrm{s}}-s A_{\mathrm{s}} \\
\frac{\mathrm{d} B_{\mathrm{i}}}{\mathrm{d} x}=\left(\mu+\frac{f}{2}+s\right) B_{\mathrm{i}}-\left(\frac{f}{2}+s\right) A_{\mathrm{i}}-\frac{f}{2} A_{\mathrm{s}} .
\end{array}\right.
$$

Soit en sommant membre à membre (I.1) et (I.3), puis (I.2) et (I.4) :

$$
\text { II }\left\{\begin{array}{l}
\frac{\mathrm{d}\left(A_{\mathrm{i}}+A_{\mathrm{s}}\right)}{\mathrm{d} x}=-\left(\mu+\frac{f}{2}+s\right)\left(A_{\mathrm{i}}+A_{\mathrm{s}}\right)+\left(\frac{f}{2}+s\right)\left(B_{\mathrm{i}}+B_{\mathrm{s}}\right) \\
\frac{\mathrm{d}\left(B_{\mathrm{i}}+B_{\mathrm{s}}\right)}{\mathrm{d} x}=\left(\mu+\frac{f}{2}+s\right)\left(B_{\mathrm{i}}+B_{\mathrm{s}}\right)-\left(\frac{f}{2}+s\right)\left(A_{\mathrm{i}}+A_{\mathrm{s}}\right) .
\end{array}\right.
$$

Soit en sommant membre à membre, puis en retranchant membre à membre (II.1) et (II.2) :

$$
\begin{aligned}
& \text { III }\left\{\begin{array}{l}
\frac{\mathrm{d}(A+B)}{\mathrm{d} x}=(\mu+f+2 s)(B-A) \\
\frac{\mathrm{d}(A-B)}{\mathrm{d} x}=-\mu(A+B)
\end{array}\right. \\
& \mathrm{IV}\left\{\begin{array}{l}
\frac{\mathrm{d}\left(A_{\mathrm{s}}+B_{\mathrm{s}}\right)}{\mathrm{d} x}=\left(\mu+\frac{f}{2}+2 s\right)\left(B_{\mathrm{s}}-A_{\mathrm{s}}\right) \\
\frac{\mathrm{d}\left(A_{\mathrm{s}}-B_{\mathrm{s}}\right)}{\mathrm{d} x}=-\left(\mu+\frac{f}{2}\right)\left(A_{\mathrm{s}}+B_{\mathrm{s}}\right) .
\end{array}\right.
\end{aligned}
$$

Tenant compte des conditions aux limites :

$$
\begin{array}{rlrl}
\text { en } x=0 & A & =\varphi_{\mathrm{t}} \\
\text { en } x=-l & B & =0 \\
& A & =\varphi_{0} \\
B & =\varphi_{\mathrm{r}} .
\end{array}
$$

La solution du système III conduit à l'expression suivante du flux total transmis :

$$
\left.\frac{\left[\varphi_{\mathrm{t}}\right]_{\mathrm{tot}}}{\varphi_{0}}=1 /\left[\cosh (l \sqrt{\mu(\mu+f+2 s)})+\frac{2 \mu+f+2 s}{2 \sqrt{\mu(\mu+f+2 s)}} \sinh (l \sqrt{\mu(\mu+f+2 s})\right)\right] .
$$

Lorsque $s=\mu=0$ on obtient la relation de Langevin :

$$
\frac{\left[\varphi_{\mathrm{t}}\right]_{\mathrm{tot}}}{\varphi_{0}}=1 /\left(1+f \frac{l}{2}\right)
$$


On obtient aisément de la même manière le flux transmis suivant la direction de $\varphi_{0}, \varphi_{\mathrm{ts}}$ :

$$
\begin{aligned}
\frac{\varphi_{\mathrm{ts}}}{\varphi_{0}}=1 /\left[\cosh \left(l \sqrt{\left(\mu+\frac{f}{2}\right)\left(\mu+\frac{f}{2}+2 s\right)}\right)+\right. & \\
& \left.+\frac{2 \mu+\frac{f}{2}+2 s}{2 \sqrt{\left(\mu+\frac{f}{2}\right)\left(\mu+\frac{f}{2}+2 s\right)}} \sinh \left(l \sqrt{\left(\mu+\frac{f}{2}\right)\left(\mu+\frac{f}{2}+2 s\right)}\right)\right] .
\end{aligned}
$$

Le flux transmis de façon isotrope est égal à la différence des flux $\left[\varphi_{\mathrm{t}}\right]_{\mathrm{tot}}$ et $\varphi_{\mathrm{ts}}$ :

$$
\varphi_{\mathrm{ti}}=\left[\varphi_{\mathrm{t}}\right]_{\mathrm{tot}}-\varphi_{\mathrm{ts}} .
$$

De la même manière on obtient le flux total rétrodiffusé $\left[\varphi_{\mathrm{r}}\right]_{\text {tot }}$ et le flux réfléchi de façon spéculaire $\varphi_{\mathrm{rs}}$ :

$$
\begin{aligned}
& r_{\mathrm{tot}}=\frac{\left[\varphi_{\mathrm{r}}\right]_{\mathrm{tot}}}{\varphi_{0}}=\frac{\frac{f+2 s}{2 \sqrt{\mu(\mu+f+2 s)}} \sinh (l \sqrt{\mu(\mu+f+2 s)})}{\frac{2 \mu+f+2 s}{2 \sqrt{\mu(\mu+f+2 s)}} \sinh (l \sqrt{\mu(\mu+f+2 s)})+\cosh (l \sqrt{\mu(\mu+f+2 s)})} \\
& r_{\mathrm{s}}=\frac{\varphi_{\mathrm{rs}}}{\varphi_{0}}= \\
& \left.\frac{s}{\sqrt{\left(\mu+\frac{f}{2}\right)\left(\mu+\frac{f}{2}+2 s\right)}} \sinh \left(l \sqrt{\left(\mu+\frac{f}{2}\right)\left(\mu+\frac{f}{2}\right.}+2 s\right)\right) . \\
& \frac{2 \mu+\frac{f}{2}+2 s}{2 \sqrt{\left(\mu+\frac{f}{2}\right)\left(\mu+\frac{f}{2}+2 s\right)}} \sinh \left(l \sqrt{\left(\mu+\frac{f}{2}\right)\left(\mu+\frac{f}{2}+2 s\right)}\right)+\cosh \left(l \sqrt{\left(\mu+\frac{f}{2}\right)\left(\mu+\frac{f}{2}+2 s\right)}\right)
\end{aligned}
$$

Le flux rétrodiffusé de façon isotrope est égal à la différence $\left[\varphi_{\mathrm{r}}\right]_{\mathrm{tot}}-\varphi_{\mathrm{rs}}=\varphi_{\mathrm{ri}}$.

Pour des valeurs assez grandes des épaisseurs optiques

$$
l \sqrt{\mu(\mu+f+2 s)} \quad \text { et } \quad l \sqrt{\left(\mu+\frac{f}{2}\right)\left(\mu+\frac{f}{2}+2 s\right)},
$$

les rapports des cosinus et sinus hyperboliques des expressions (4) et (5) tendent vers l'unité.

$$
\begin{gathered}
r_{\mathrm{tot}}=\frac{\left[\varphi_{\mathrm{r}}\right]_{\mathrm{tot}}}{\varphi_{0}}=\frac{f+2 s}{2 \mu+f+2 s+2 \sqrt{\mu(\mu+f+2 s)}} \\
r_{\mathrm{s}}=\frac{\varphi_{\mathrm{rs}}}{\varphi_{0}}=\frac{2 s}{2 \mu+f+2 s+2 \sqrt{\left(\mu+\frac{f}{2}\right)\left(\mu+\frac{f}{2}+2 s\right)}} .
\end{gathered}
$$

A cause du couplage des équations du système I, le flux diffusé dans les directions autres que celle de la réflexion spéculaire dépend du flux réfléchi spéculairement. Nous pouvons exprimer la variation du flux rétrodiffusé de façon isotrope lorsque les particules sont orientées de façon aléatoire et lorsqu'elles sont orientées suivant une direction donnée (par exemple sous l'effet d'une contrainte hydrodynamique) :

$$
\Delta \varphi_{\mathrm{i}}=\left[\left[\varphi_{\mathrm{r}}\right]_{\mathrm{tot}}-\varphi_{\mathrm{rs}}\right]_{s=0}-\left[\left[\varphi_{\mathrm{r}}\right]_{\mathrm{tot}}-\varphi_{\mathrm{rs}}\right]_{s=s_{\max }} .
$$

Pour une particule de taille importante $(d>\lambda)$ les mécanismes responsables de la diffusion de la lumière sont :

a) la diffraction par les bords de la particule;

b) la réflexion sur sa surface;

c) la réfraction à travers son volume [12]. 
Soient $N_{\mathrm{i}}$ le nombre de particules orientées de façon aléatoire, $N_{\mathrm{s}}$ le nombre de particules orientées suivant une direction donnée et $N_{0}$ le nombre total de particules, par unité de volume :

$$
N_{0}=N_{\mathrm{i}}+N_{\mathrm{s}} \text {. }
$$

Dans une hypothèse de flux diffusé de façon parfaitement isotrope, nous pouvons poser :

$$
f=R N_{\mathrm{i}} \sigma_{\mathrm{i}}
$$

(où $\sigma_{\mathrm{i}}$ est la surface réelle de la particule)

$$
s=R^{\prime} N_{\mathrm{s}} \sigma_{\mathrm{s}}
$$

(où $\sigma_{\mathrm{s}}$ est une fraction de la surface de la particule susceptible de réfléchir la lumière dans une direction donnée).

$R$ et $R^{\prime}$ sont fonction des indices des milieux constituant la particule et le milieu suspendant

$$
\mu=\beta N_{0} v
$$

(où $v$ est le volume particulaire).

En introduisant les expressions de $f, s$, et $\mu$ dans (6) et (7), nous traiterons le cas particulier $s=0$.

Pour des particules dont l'orientation est aléatoire $\left(s=0, N_{\mathrm{i}}=N_{0}\right)$ le coefficient de réflexion $r$ :

$$
r=\frac{R \sigma_{\mathrm{i}}}{2 \beta v+R \sigma_{\mathrm{i}}+2 \sqrt{\beta v\left(\beta v+R \sigma_{\mathrm{i}}\right)}}
$$

est indépendant de la concentration. Ce résultat peut sembler paradoxal, en fait ceci n'est exact que si l'épaisseur de la suspension est supérieure à une longueur critique qui, elle, est fonction de la concentration. Quand les particules élémentaires s'agrègent pour former $N_{\mathrm{i}}^{\prime}$ agrégats de surface $\sigma_{\mathrm{i}}^{\prime}$ :

$$
f^{\prime}=R N_{\mathrm{i}}^{\prime} \sigma_{\mathrm{i}}^{\prime} \text {. }
$$

Soit $m$ le nombre de particules par agrégat :

$$
\begin{gathered}
N_{\mathrm{i}}^{\prime}=N_{0} m^{-1} \\
r^{\prime}=\frac{R N_{\mathrm{i}}^{\prime} \sigma_{\mathrm{i}}^{\prime}}{2 \beta N_{0} v+R N_{\mathrm{i}}^{\prime} \sigma_{\mathrm{i}}^{\prime}+2 \sqrt{\beta N_{0} v\left(\beta N_{0} v+R N_{\mathrm{i}}^{\prime} \sigma_{\mathrm{i}}^{\prime}\right)}} .
\end{gathered}
$$

Soient $\sigma$ et $\sigma^{\prime}$ les aires totales des particules désagrégées et des particules agrégées par unité de volume; en résolvant (9) et (10) par rapport à $\sigma$ et $\sigma^{\prime}$, on obtient :

$$
\sigma=\frac{4 \beta N_{0} v}{R} \frac{r}{(1-r)^{2}} ; \quad \sigma^{\prime}=\frac{4 \beta N_{0} v}{R} \frac{r^{\prime}}{\left(1-r^{\prime}\right)^{2}}
$$

d'où :

$$
\frac{\sigma^{\prime}}{\sigma}=\frac{r^{\prime}}{r}\left(\frac{1-r}{1-r^{\prime}}\right)^{2}
$$

1.2 Application AU CAS D'UNE SUSPENSION SANGUINE. - Les hématies du sang humain normal s'agrègent pour former des rouleaux. Soit $N_{0}$ le nombre d'hématies et le nombre $n(m)$ de rouleaux de $m$ hématies par unité de volume.

Considérons le modèle d'agrégat suivant : les hématies sont des disques d'épaisseur $b$ de rayon $a$ empilés, formant des cylindres de longueur $m b$. Soit $p=m-1$, le paramètre caractérisant les agrégats, $n(p) \mathrm{d} p$ le nombre de rouleaux par unité de volume dont le paramètre $p$ est compris entre $p$ et $p+\mathrm{d} p$. Le nombre total d'agrégats par unité de volume est :

$$
N=\int_{0}^{\infty} n(p) \mathrm{d} p .
$$

Le nombre total de particules par unité de volume est :

$$
N_{0}=\int_{0}^{\infty} p n(p) \mathrm{d} p .
$$


Enfin, les valeurs moyennes de $p$ et de $m$ sont :

$$
\begin{aligned}
&\langle p\rangle= \frac{\int_{0}^{\infty} p n(p) \mathrm{d} p}{\int_{0}^{\infty} n(p) \mathrm{d} p}=\frac{N_{0}}{N} \\
&\langle m\rangle=\langle p\rangle+1 .
\end{aligned}
$$

On en déduit les valeurs moyennes de $\sigma^{\prime}$ et $\sigma$ :

$$
\begin{aligned}
& \frac{\left\langle\sigma^{\prime}\right\rangle}{\langle\sigma\rangle}=\frac{\frac{\alpha^{\prime}}{m}+1}{\alpha^{\prime}+1} \\
& \alpha=\frac{a}{b}=2 \\
& \alpha^{\prime}=\frac{a^{\prime}}{b^{\prime}}=2,63 \text {. }
\end{aligned}
$$

Pour des globules agrégés du sang humain normal le rapport $\alpha^{\prime}$ est égal à 2,63 $\left({ }^{1}\right)$. Ainsi :

$$
\left(\frac{1-r}{1-r^{\prime}}\right)^{2} \frac{r^{\prime}}{r}=\frac{1}{3,63}\left(\frac{2,63}{\langle m\rangle}+1\right) \text {. }
$$

On constate qu'il est nécessaire de se donner un modèle géométrique de l'agrégat pour relier les flux rétrodiffusés à l'aire des agrégats. Sur la figure 3 nous avons tracé le rapport : pouvoir réflecteur de la suspension agrégée/pouvoir réflecteur de la suspension non agrégée, en fonction du nombre $m$ de particules par agrégat pour des agrégats cylindriques et sphériques. On constate que pour des agrégats formés de plus de dix particules le pouvoir réflecteur varie peu, ce qui limite la précision des résultats dans ce domaine.

2. Dispositif expérimental. - Les principaux éléments du montage sont indiqués sur la figure 4. Un laser HeNe $(\lambda=6328 \AA)$ Spectra Physics Mode 143, éclaire un échantillon de sang contenu dans une cuve cylindrique transparente en rotation dans laquelle plonge un cylindre coaxial, stator d'un viscosimètre de type Couette (Contraves LS 30). L'échantillon de sang contenu dans l'entrefer $(\Delta R=0,5 \mathrm{~mm})$ des deux cylindres peut être soumis à des taux de cisaillement $\dot{\gamma}$ contrôlés variant de $1,7 \times 10^{-2} \mathrm{~s}^{-1}$ à $128,5 \mathrm{~s}^{-1}$. Une lentille $\mathrm{L}$ focalise le flux lumineux rétrodiffusé dans une direction faisant un angle $\theta$ avec le faisceau incident. L'angle $\theta$ est choisi de telle façon que la réflexion spéculaire ne soit pas détectée; ainsi, le flux lumineux détecté est égal à la fraction $\mathrm{d} \Omega / 2 \pi$ du flux lumineux total rétrodiffusé de façon isotrope ( $\mathrm{d} \Omega$ étant l'angle solide correspondant à l'ouverture de $\mathrm{L}$ ) ; un détecteur photométrique linéaire est placé au foyer de $\mathrm{L}$ (photo-élément Siemens BFN 33); le signal électrique du détecteur est enre-

$\left({ }^{1}\right)$ Pour des globules non agrégés le rapport $\alpha$ est un peu inférieur à 2. Pour des globules agrégés, les dimensions sont estimées en considérant les rouleaux vus de côté (par exemple dans [13]).

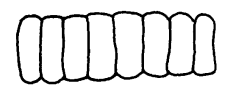

(1)

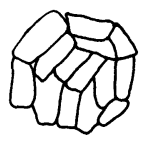

(2)

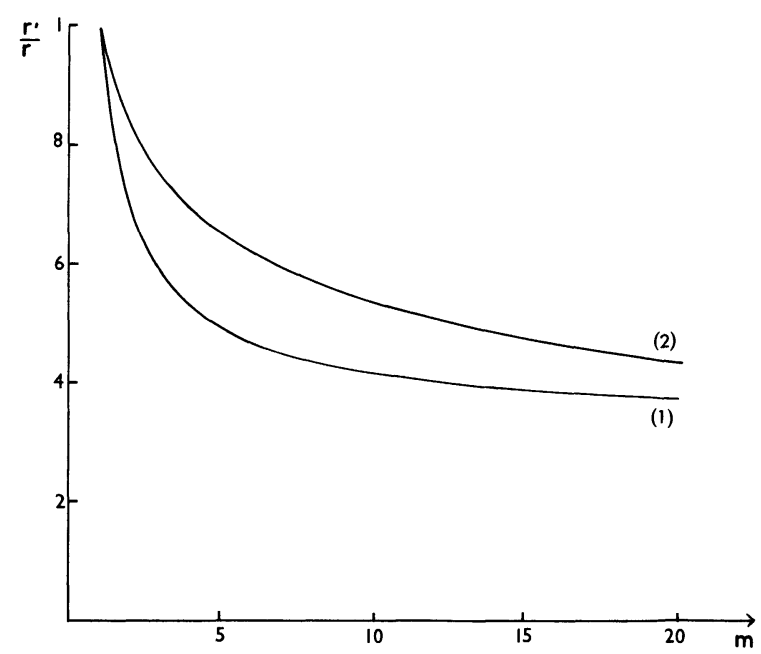

Fig. 3. - Réflectivité de la suspension en fonction du nombre de particules $m$ constituant un agrégat ((1) agrégat cylindrique, (2) agrégat sphéroïde).

[Suspension reflectivity versus the $m$ number of particles per aggregate ((1) cylindrical aggregate, (2) spheroid aggregate).]

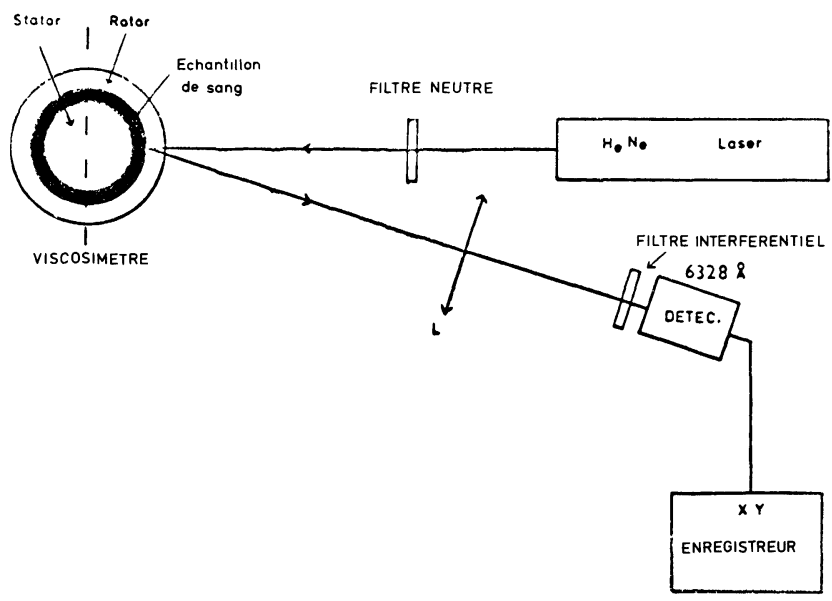

Fig. 4. - Représentation schématique du dispositif expérimental.

[Schematic view of experimental apparatus.]

gistré sur une table traçante. Une série de filtres absorbants neutres et une cuve remplie de billes de verre (diamètre $=50 \mu$ ) constituant une suspension blanche permettent d'étalonner le détecteur. Un filtre interférentiel placé devant le détecteur élimine les flux lumineux parasites.

3. Résultats expérimentaux.

3.1 Cinétique D'AGRÉGATION $\grave{A} \dot{i}=0$. - Le montage décrit ci-dessus permet de déterminer la taille moyenne des agrégats 
en fonction du temps, partant d'un état où les hématies sont totalement dissociées. La dissociation complète est obtenue en soumettant la suspension à une contrainte hydrodynamique suffisante : par exemple, pour un échantillon de sang normal un cisaillement de $60 \mathrm{~s}^{-1}$ est suffisant. Une courbe caractéristique représentant le flux lumineux rétrodiffusé est tracée sur la figure 5. L'analyse de cette courbe montre :

La région $a$ ). - La partie du flux rétrodiffusé de façon isotrope par les globules dissociés et orientés dans le champ de cisaillement est plus faible que lorsque les globules sont dissociés et désorientés; en effet, lorsque les particules sont orientées une partie importante du flux incident est réfléchie spéculairement et le flux isotrope diminue d'autant.

La région $b$ ). - Après l'arrêt du cylindre extérieur du Couette, le pic correspond à la lumière diffusée de façon isotrope par les globules dissociés et désorientés.

La région $c$ ). - Le flux rétrodiffusé diminue lors de la formation des rouleaux.

Analysons de façon plus détaillée le passage de la région $a$ ) à la région $b$ ); la suspension contient des particules orientées, dans le champ de cisaillement qu'on appelle particules $\mathrm{A}$ et des particules non orientées qui peuvent être des particules isolées ou des multiplets, qu'on appelle particules $B$. La fréquence de collision d'une particule A avec les particules B est $\lceil 14\rceil$ :

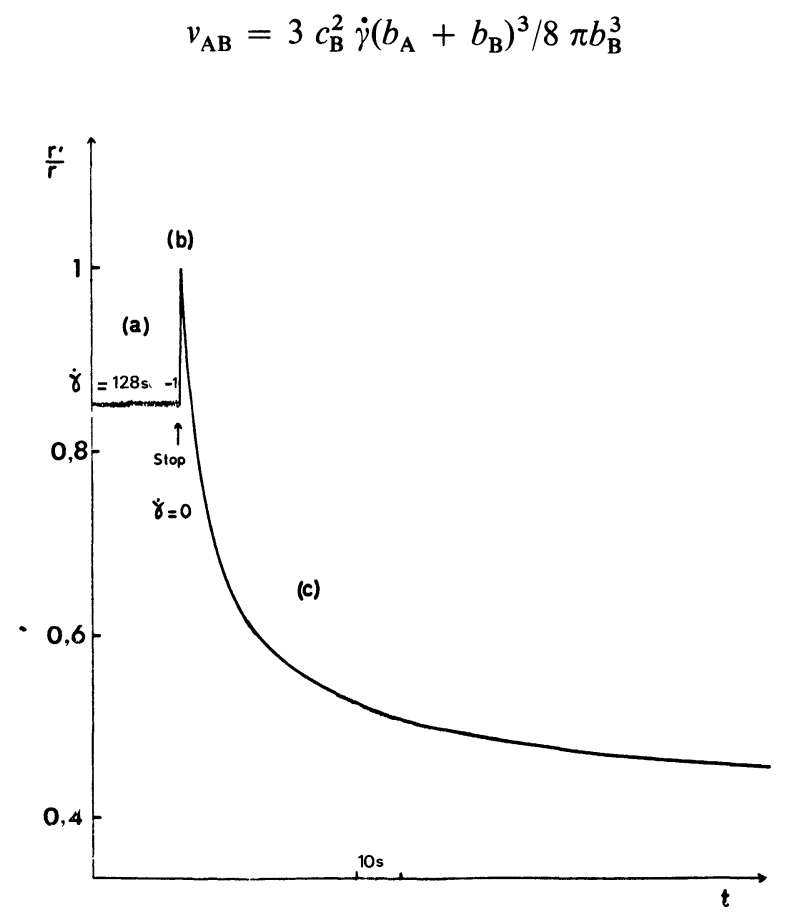

Fig. 5. - Variation de la réflectivité en fonction du temps (échelle $10 \mathrm{~s} / \mathrm{cm}$ ). Sang humain normal. La différence $\Delta r$ entre l'état d'équilibre $\dot{\gamma}$ et l'état correspondant à une orientation aléatoire est proportionnelle à $r_{\mathrm{s}}\left(\mathrm{ici} \dot{\gamma}=128 \mathrm{~s}^{-1}\right)$.

[Reflectivity variation with time (scale $10 \mathrm{~s} / \mathrm{cm}$ ). Normal human blood. The difference $\Delta r$ between the equilibrium state at $\dot{\gamma}$ and the random state is proportional to $r_{\mathrm{s}}$ (here $\dot{\gamma}=128 \mathrm{~s}^{-1}$ ).] où $b_{\mathrm{A}}, b_{\mathrm{B}}, c_{\mathrm{B}}$ sont respectivement les rayons des particules $\mathrm{A}, \mathrm{B}$ et la concentration des particules $\mathrm{B}$.

Un ordre de grandeur du temps de désorientation des particules orientées peut être obtenu en estimant $v_{\mathrm{AB}}^{-1}$ pour une concentration $c_{\mathrm{B}}=0,4, \bar{\gamma}=50 \mathrm{~s}^{-1}$, $b_{\mathrm{A}} \neq b_{\mathrm{B}}$. Ainsi,

$$
\tau_{\mathrm{AB}}=v_{\mathrm{AB}}^{-1}=0,13 \mathrm{~s} .
$$

On peut donc considérer que pendant le temps de diffusion d'un échelon de cisaillement, de l'ordre de $\rho L^{2} / \eta=0,2$ s (où $L$ est la distance entre les deux cylindres du Couette, $\eta$ la viscosité, $\rho$ la densité du fluide), les collisions entre particules rétablissent l'isotropie des orientations.

Notons que le temps de désorientation des particules par diffusion brownienne est très supérieur à $\tau_{\mathrm{AB}}$ puisque :

$$
\tau_{\mathrm{R}}=\mathscr{D}_{\mathrm{rot}}^{-1}=\frac{8 \pi \eta_{0} a^{3}}{k T} \simeq 10^{3} \mathrm{~s}
$$

pour des particules sphériques dans un plasma. Ce temps est encore supérieur pour des particules allongées. Sur la figure 6 la région $b$ ) a été tracée avec une échelle de temps dilatée, pour différents taux de cisaillement initiaux. Le temps de désorientation expérimental est de $0,1 \mathrm{~s}$. Les effets dus à l'orientation des globules décroissent avec le taux de cisaillement et deviennent négligeables pour $\dot{\gamma}=6 \mathrm{~s}^{-1}$. La hauteur du pic correspondant à la différence entre état orienté
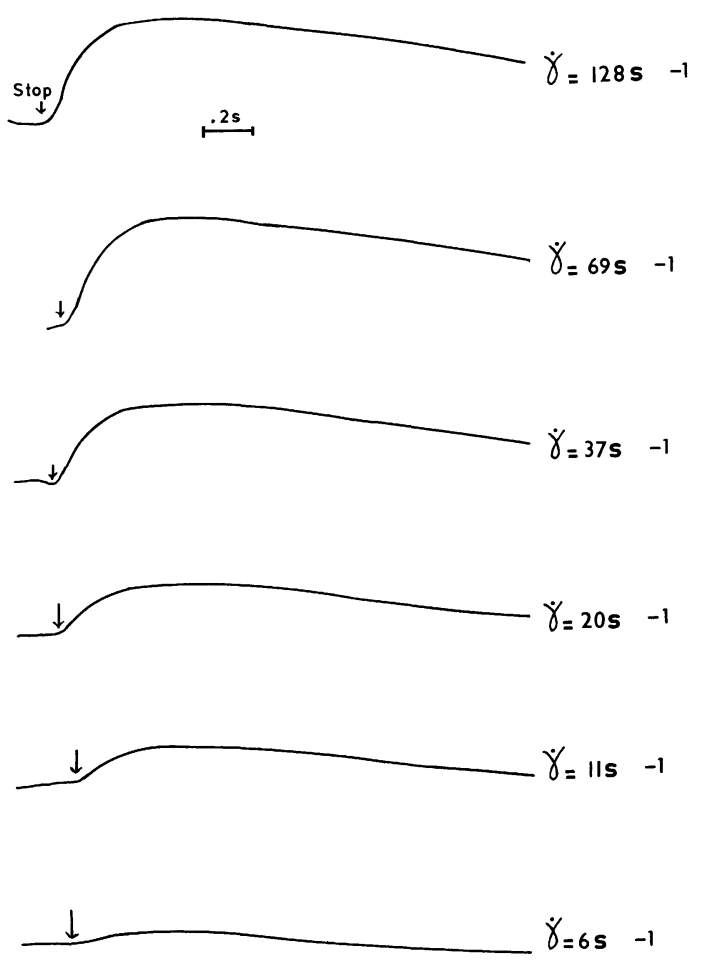

Fig. 6. - Signaux transitoires correspondant à la désorientation des particules pour différentes valeurs du taux de cisaillement initial (échelle $0,2 \mathrm{~s} / \mathrm{cm}$ ).

[Desorientation transient signals for several starting shear rate values (scale $0.2 \mathrm{~s} / \mathrm{cm}$ ).] 
et désorienté des particules dépend de la concentration. Nous avons montré dans la partie théorique que le signal correspondant à l'état désagrégé et orienté ne dépend pas de la concentration, par contre le nombre de particules participant à la réflexion spéculaire croît avec la concentration. Cette dépendance a été mise en évidence expérimentalement pour une suspension sanguine normale et pour une suspension d'hématies lavées trois fois dans du sérum physiologique (hématies n'agrégeant pas) (Fig. 7).

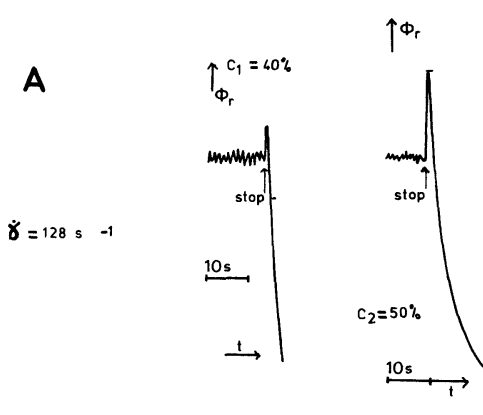

B
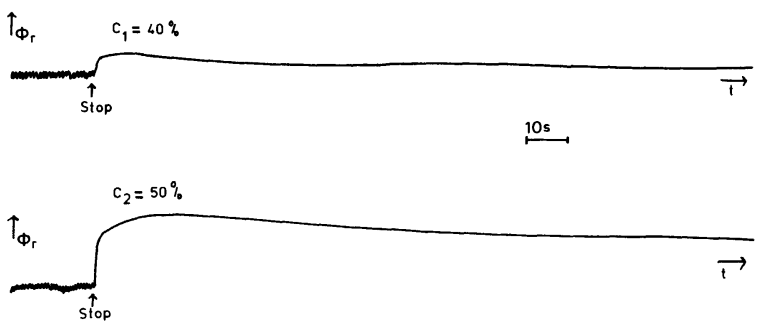

Fig. 7. - Variation de la réflectivité en fonction du temps pour deux concentrations ( $c=0,4, c=0,5)$. A) Sang normal. B) Globules lavés trois fois dans du sérum physiologique.

[Reflectivity variation with time for two suspension concentrations $(c=0.4, c=0.5)$. A) Normal blood. B) Three times washed cells in normal saline solution.]

Enfin, l'étude de la région $c$ ) de la courbe de la figure 5 permet de décrire la cinétique d'agrégation des particules. Pour étudier cette région, nous fixons l'origine des temps $t$ au maximum du pic de $r^{\prime} / r$; au temps $t=0, r^{\prime} / r=1$. Si nous traçons la variation de $\log \left[\frac{\mathrm{d} r^{\prime} / r}{\mathrm{~d} t}\right]$ en fonction du temps on obtient pour $0 \leqslant t \leqslant 20 \mathrm{~s}$ une droite $\Delta$. L'équation (12) montre qu'au voisinage de $t=0, r \simeq r^{\prime}$ :

$$
\frac{r^{\prime}}{r} \simeq \frac{1}{3,63}\left(\frac{2,63}{\langle m\rangle}+1\right)
$$

d'où :

$$
\log \left[\frac{\mathrm{d}}{\mathrm{d} t}\left(\frac{r^{\prime}}{r}\right)\right] \# \log \frac{2,63}{3,63}-\log \left[\frac{\mathrm{d}\langle m\rangle}{\mathrm{d} t}\right]
$$

dans l'intervalle $/ 0,20 \mathrm{~s}$ la variation de $\langle m\rangle$ en fonction du temps est exponentielle; la pente de la droite
$\Delta$ est égale à l'inverse du temps caractéristique d'agrégation $\tau_{\mathrm{a}}$. Pour la plupart des sangs normaux ( $c=0,45) \tau_{\mathrm{a}}$ est compris entre $4 \mathrm{~s}$ et $6 \mathrm{~s}$ (Tableau I). L'addition de drogues telles que les Dextranes de haut poids moléculaire provoque une agrégation plus rapide $\tau_{\mathrm{a}}<4 \mathrm{~s}$; par contre l'addition de $\mathrm{NaSa}$ a un effet désagrégeant $\tau_{\mathrm{a}}>4 \mathrm{~s}$.

Pour $t>20 \mathrm{~s}$ la croissance du nombre de particules par agrégat n'est plus exponentielle.

Tableau I

$$
\begin{array}{cccc} 
& \begin{array}{c}
\text { sang } \\
\text { désagrégé }
\end{array} & \begin{array}{c}
\text { sang normal } \\
\text { agrégé }
\end{array} & \begin{array}{c}
\text { sang }+\mathrm{D}_{\mathrm{x}} 500 \\
2,5 \mathrm{~g} \%
\end{array} \\
r, r^{\prime} & r=0,14 & r^{\prime}=0,05 & r^{\prime}=0,03 \\
\tau_{\mathrm{a}} & \tau_{\mathrm{a}}=\infty & \tau_{\mathrm{a}}=5 \mathrm{~s} & \tau_{\mathrm{a}}=1,5 \mathrm{~s}
\end{array}
$$

\subsection{TAILLE MOYENNE DES AGRÉGATS DANS UN ÉCOU-} LEMENT DE COUETTE. - Nous avons mesuré la lumière rétrodiffusée par du sang complet placé dans une cuve cylindrique transparente contenant le cylindre extérieur d'un viscosimètre de type Couette (LS 30 Contraves). Pour chaque valeur du taux de cisaillement moyen $\dot{\gamma}$ dans le volume de mesure le flux rétrodiffusé est mesuré après qu'un équilibre dynamique soit établi. En arrêtant très rapidement le cylindre extérieur on peut mesurer le flux rétrodiffusé correspondant à la diffusion isotrope du flux incident $\varphi_{0}$ par les agrégats de taille $m=m(\dot{\gamma})$.

Au-delà d'une contrainte $\theta_{\mathrm{D}}=\eta_{0} \dot{\gamma}_{\mathrm{D}}$, les agrégats sont complètement dissociés, le flux rétrodiffusé correspondant aux particules orientées de façon aléatoire est constant; par contre la partie isotrope du flux rétrodiffusé correspondant à l'équilibre dynamique dans un champ de cisaillement constant $\dot{\gamma}$ passe par un maximum et diminue lorsque les globules s'orientent dans l'écoulement, figure 8 .

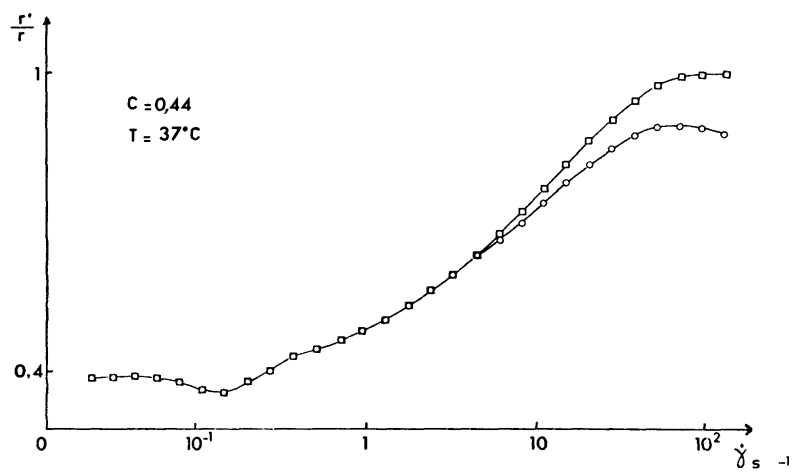

Fig. 8. - Variation de la réflectivité en fonction du taux de cisaillement : - —-état d'équilibre dynamique à $\dot{\gamma}:-\square-$ état d'orientation aléatoire. On remarque un minimum au voisinage de $\dot{\gamma}=10^{-1} \mathrm{~s}^{-1}$ qui correspond peut-être à un changement de structure des agrégats.

[Reflectivity variation with shear rate : - - - dynamical equilibrium state at $\dot{\gamma} ;-\square-$ random orientation state. Note the minimum near $\dot{\gamma}=10^{-1} \mathrm{~s}^{-1}$ which is perhaps connected to aggregate structure change.] 
Il n'existe pas, à notre connaissance, d'étude théorique de la cinétique d'agrégation des globules rouges (à part les approches de Casson et Murata [16]). Des observations visuelles directes [17] ayant montré que la taille des agrégats est une fonction décroissante de la contrainte, nous proposons, pour une concentration et une viscosité du milieu suspendant fixées $\left(\eta_{0}\right.$, viscosité du plasma) une loi simple reliant la taille moyenne $m$ des agrégats au taux de cisaillement moyen $\dot{\gamma}$ :

$$
m=\left(\frac{\dot{\gamma}_{\mathrm{D}}}{\dot{\gamma}}\right)^{n}
$$

où $n$ est un exposant que nous déterminons expérimentalement. En portant cette expression dans (12), on obtient :

$$
\frac{r^{\prime}}{r} \cdot\left(\frac{1-r}{1-r^{\prime}}\right)^{2}=\frac{1}{3,63}\left[2,63\left(\frac{\dot{\gamma}}{\dot{\gamma}_{\mathrm{D}}}\right)^{n}+1\right]
$$

ou encore :

$$
\begin{array}{r}
\log \left[\frac{1}{2,63}\left[3,63 \frac{r^{\prime}}{r}\left(\frac{1-r}{1-r^{\prime}}\right)^{2}-1\right]\right]= \\
=n \log \dot{\gamma}-n \log \dot{\gamma}_{\mathrm{D}} .
\end{array}
$$

En déterminant à partir des résultats expérimentaux $r^{\prime}(\dot{\gamma})$, l'expression du premier membre de (15) en fonction du logarithme du cisaillement, on détermine par une régression linéaire l'exposant $n$ et le cisaillement critique $\dot{\gamma}_{\mathrm{D}}$ (Fig. 9). Pour les sangs normaux

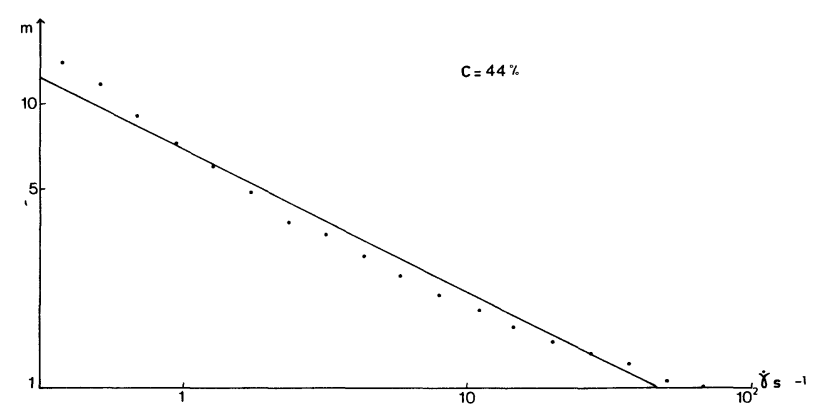

Fig. 9. - Variation du nombre moyen de particules par agrégat en fonction du taux de cisaillement.

[Particles mean number versus shear rate.] nous avons trouvé $n$ voisin de 0,5 ; le cisaillement critique $\dot{\gamma}_{\mathbf{D}}$ varie avec la concentration et la viscosité du milieu suspendant. Il décroît quand on augmente la concentration ou la viscosité du plasma. Pour des sangs normaux et une concentration de $0,4, \dot{\gamma}_{D}$ est compris entre $50 \mathrm{~s}^{-1}$ et $60 \mathrm{~s}^{-1}$.

4. Conclusion. - Nous avons résumé dans le tableau ci-après, les paramètres caractéristiques de l'agrégation érythrocytaire pouvant être déterminés à partir du signal de rétrodiffusion.

\section{Tableau II}

$$
\begin{aligned}
& \text { Paramètres caractéristiques Méthode } \\
& m(0)=\text { nombre moyen de glo- Mesure du pouvoir réflecteur } r^{\prime}
\end{aligned}
$$

Le paramètre suivant n'a pu être déterminé avec notre appareil faute de pouvoir réaliser un échelon entre deux valeurs données du cisaillement.
$\tau(\dot{\gamma})=$ temps d'agrégation ou Analyse temporelle du signal de désagrégation pour $\frac{r^{\prime}}{r}=f(t, \dot{\gamma})$ ment

La détermination expérimentale de ces paramètres est d'un grand intérêt pour la caractérisation en milieu hospitalier des états d'agrégation pathologique du sang humain [10]. La quantification de ces résultats nous a permis d'entreprendre une étude théorique de la cinétique d'agrégation des globules rouges, étude qui est en cours.

\section{Bibliographie}

[1] Kurodo, K., Jujino, M., On the cause of increases in light transparency of erythrocyte suspensions in flow. Biorheology 1 (1963) 167-182.

[2] Yanami, Y., Intagliatta, M., Frasher, W. G., Wayland, H., Photometric study of erythrocytes in shear flow. Biorheology 2 (1964) 165-168.
[3] JANSONIUS, N. J., ZuJLSTRA, W. G., Various factors influencing rouleaux formation of erythrocytes, studied with the aid of syllectometry. Proc. Kon. Ned. Akad. Wet. Ser. C 68 (1965) 122-127.

[4] Dognon, A., Suquet, P., Facteur de diffusion diffuse des suspensions de particules colorées. J. Chim. Phys. 10 (1957) 815-826. 
[5] Healy, J. C., Thèse, Université Paris VI, Paris (1973).

[6] Klose, H. J., Volger, E., Brechtelsbauer, H., Heinich, L. SCHMid-SchonBeIN, H., Microrheology and light transmission of blood. Pflügers Arch. 333 (1972) 126-139.

[7] Usami, S., ChiEN, S., Optical reflectometry of red blood cell aggregation under shear-flow. Proc. VIIth Conference on microcirculation (Aberdeen 1972) 91-98, Basel : Karger (1973).

[8] Mills, P., Adler, P., Quemada, D., Dufaux, J., Etude de l'agrégation de particules agrégeables par rétrodiffusion laser. Journal des maladies cardiovasculaires, Paris $\mathbf{4}$ (1979) 91-94.

[9] Dognon, A., Granulométrie optique de la suspension sanguine. Nouvelle technique d'étude. C. R. Hebd. Séan. Acad. Sci. D 268 (1969) 974-975.

[10] Mills, P., Quemada, D., Dufaux, J., Red blood cell aggregation and orientation studies by laser light back scattering. Hémorhéologie et Pathologie. Symp. Nancy Oct. (1979).
[11] Schuster, J., Radiation through a foggy atmosphere. Astrophys. J. (1905).

[12] KASTLER, A., La diffusion de la lumière par les milieux troubles, Actualités scientifiques et industrielles (Herman Edit.) Paris, 1952.

[13] Chien, S., Usami, S., Dellenback, R. J. and Bryant, C. A., Comparative hemorheology, hematological implications of species differences in blood viscosity. Biorheology 8 (1971) 35-57.

[14] Goldsmith, H. L., Mason, S. G., in R. F. Eirich Edit., Rheology, Theory and Applications, Vol. 4 (Acad. Press, New York and London) 1967, p. 172.

[15] Casson, N., in C. C. Mill Edit., Rheology of Disperse Systems (Pergamon Press, London) 1959, p. 84.

[16] Murata, T., Theory of non-newtonian viscosity of blood at low shear-rate. Effect of rouleaux. Biorheology 13 (1976) 287-296.

[17] Schmid-Schonbein, H., Wells, R. E., Schildkraut, R., Microscopy and viscosimetry of blood flowing under uniform shear rate (rheoscopy). J. Appl. Phys. 26 (1969) 674-678. 